\title{
The Spin is the Origin of the Charge on a Charged Particle
}

\author{
Salah Eid \\ Faculty of Arts - Suez Canal University - Ismailia - EGYPT
}

\begin{abstract}
The charge on a charged particle is the energy created by its revolution around its axis or its spin, this explains the charge on the electron or the proton. It is well known that the two energies or charges of these two particles are equal in magnitude and opposite in direction or sign, why? because the ratio between electron's revolution squared velocity and proton's revolution one is the same as the ratio between the mass $m_{p}$ of the proton measuring to the mass of electron $m_{e}$, thus each has the same energy or charge. In the same time, these two charges are the equal and opposite halves of the circular motion of the electron around the proton in the hydrogen atom, the smallest and basic astronomical system in the universe.
\end{abstract}

DOI: $10.7176 /$ APTA/80-05

Publication date:October $31^{\text {st }} 2019$

1- The spin of the particle as the source of charge :

No escape from considering the spin of the particle is the source of its charge or energy. We are going to prove this by our unifying constant $U$

$$
m v^{2} r=\frac{e^{2}}{4 \pi \varepsilon_{0}}=2.30 \times 10^{-28} J-m^{(1)}
$$

Now, Dividing U or $2.30 \times p 10^{-28} \mathrm{~J}-m$ by the mass of electron in $\mathrm{Kg}$, we have the electron's revolution squared velocity at radius $r$

$$
v^{2} r=252.46
$$

Dividing $\mathrm{U}$ by the mass of the proton in $\mathrm{Kg}$, we have the proton's revolution squared velocity at radius $\mathrm{r}$

$$
v^{2} \mathrm{r}=0.13772
$$

The ratio between $2 \& 3$ is $\quad \frac{252.46}{0.13772}=1833.07$

And this is the same ratio between the mass of the proton to the mass of electron, where

$$
\frac{1.67 \times 10^{-27} \mathrm{Kg}}{9.11 \times 10^{-31} \mathrm{Kg}}=1833.15
$$

Therefore, $\left(m v^{2} r\right)$ the electron and proton or their charges is the same, where simply the electron moves faster than the proton but in the opposite direction or sigh.

The charge for each of them considering its mass is obtained from constant $U$ as follows

$$
m_{e} v^{2} r 4 \pi \varepsilon_{0=} m_{p} v^{2} r 4 \pi \varepsilon_{0}=e^{2}
$$

Again, the spin of the electron or proton is the source of its charge, and this charge is in the same time one of the two equal and opposite halves of the circular motion of the electron and proton in hydrogen atom.

\section{2- The Two rigid balls}

We here deal with the electron and proton as two different rigid balls. The electron is not at all a point with no measurable size, and the proton is not at all composed of quarks and gluons where its spin must be determined by the orbital angular momentum of its constituents. ${ }^{(2)}$

Tremendous efforts in CERN had been made since the late 1940s and 1950s up till now to prove that hadrons including protons and other particles are composed of quarks held together by the strong force ( as atoms and molecules are held together by the electromagnetic force). Experimenters used collider which is a particle accelerator with two directed beams of particles, they accelerated particles to relatively high kinetic energy letting them impact other particles. Large Hadrons Collider (LHC) is the world's largest and most powerful particle collider built by the European Organization for nuclear research. Over 10,000 scientists and hundreds of universities and liberators, as well as 100 countries spent their efforts in a tunnel of 27 kilometers in circumference and as deep as 175 meters beneath France-Switzerland border near Geneva ${ }^{(3)}$

Up till now with all these extensive experiments and great efforts no sign appeared proving that proton is composed of smaller constitutes like quarks, or that the well known fundamental charge could be divided into smaller parts!

Conclusion

1- The charge on a charged particle is the result of its revolution around its axis or spin. The charge on the electron equals that of the proton because the energy of each of them $\left(m v^{2} r\right)$ is the same but in opposite direction, and in the same time it is one of the two equal and opposite halves of the circular motion of the electron around the proton in hydrogen atom. 
2- We deal with the electron and proton as two different rigid balls, the electron is not at all a point without dimensions, the proton is not made up of any smaller constituents or the so called quarks or gluons.

\section{References}

1- Eid Salah, The Unifying Constant U, Advances in Physics Theories and Applications, Vol 36 (2014)

2- http:// www.quora.com/protons-h

3- http://en.m.wikipedia.org/wiki/large- Hadron-collide 